\title{
Exploring the microbiome's potential role in severe COVID-19: possible implications for prevention and treatment
}

\author{
Joyce Waterhouse ${ }^{1}$ \\ ${ }^{1}$ Affiliation not available
}

April 28, 2020

\begin{abstract}
The human microbiome, especially the microbiomes of the gastrointestinal and respiratory tracts, are potentially important in determining susceptibility to COVID-19 and the immunopathology that leads to severe disease. Data is beginning to be gathered on the risk factors for severe disease in the coronavirus disease of 2019 (COVID-19). This data will be discussed in connection with some highlights of what is being learned about the human microbiome and its relationship with viral illnesses and inflammation-related chronic diseases. In particular, possible roles for diet, lifestyle, and microbiota manipulation as means of reducing rates of severe viral disease will be explored. Some potential pharmaceutical approaches to treating severe COVID-19 disease, involving the microbiome, mast cells and hypersensitivity responses, are also discussed. It is proposed that chronic low-grade infections and/or dysbiosis may underlie the age-related diseases that are risk factors for severe disease from SARS-CoV-2 (severe acute respiratory syndrome coronavirus 2). It is also suggested that the connection between these chronic diseases and COVID-19 may have implications for understanding some of the reasons for the severity of COVID-19 in a proportion of patients. Whether or not the hypothesis of a causal role for dysbiosis or prior infection is correct, some of the suggested treatment approaches might still be worth investigating.
\end{abstract}

Keywords: microbiome, COVID-19, Westernized diet, exercise, stress, mast cells, cytokine storm, IL-6, plant-based diet, inflammation, dysbiosis

\section{Introduction}

Researchers are rapidly gaining knowledge about COVID-19 to help address the current global pandemic, with a focus on treatment and prevention of the spread of the disease[1-3]. This article has two primary aims. The first aim is to examine the characteristics of the disease and the individuals who are most susceptible to severe disease to see if they can help reveal how humans can become less susceptible. A second aim is to explore whether these considerations might suggest treatment approaches that have potential to help at least some of those who are already suffering from severe disease. It is hoped that this review will be able to suggest areas of research that could be helpful in dealing with both the current pandemic and with other similar diseases or future epidemics.

The emphasis here will be on the human microbiome and the diet, lifestyle and medical intervention factors that often affect it. This emphasis arises from the increasing research showing the profound impact of the human microbiome on immune function and many aspects of diverse disease processes[4,5]. The human 
genetic makeup is certainly important; however, the microbiotas inhabiting different parts of the human body are increasingly being shown to be crucial factors. It has been estimated that there are as many bacterial cells in the human body as human cells[6], and numerous microbial metabolites from the microbiome reach the blood stream and are increasingly being investigated[7]. This has led to the human microbiome being called the second genome[8]. One advantage of focusing on this second genome is that studies are showing that it can be altered much more easily than the human genome, apparently with beneficial effects, such as in the treatment of antibiotic-associated diarrhea due to Clostridiodes dificile infection using fecal microbiota transplants[9].

The rapid changes in diet, lifestyle, environmental exposures and medical interventions in the last 75 years has led to changes in the human microbiome that may not be optimally compatible with our evolved immune responses to pathogens. This perspective is closely related to the hygiene hypothesis[10] and its newer forms, such as the altered microflora hypothesis[11] and an extension of these earlier hypotheses that focuses on the potentially pathogenic microbes within the post-hunter-gatherer era microbiota (PHM)[12]. Throughout this review, attention will be paid to factors that might lead to the establishment of these potentially pathogenic microbes, which include environmental microbes that are less coevolved with their human hosts and thus could have greater immune suppressing/dysregulating potential.

\section{The human microbiome is affected by changing diet, lifestyle, envi- ronment and medical interventions}

SARS-CoV-2 is part of a family of coronaviruses that cause a wide spectrum of disease, from the common cold to the highly pathogenic severe acute respiratory syndrome and Middle East respiratory syndrome. A large part of the reason that SARS-CoV-2 is so problematic is its broad range of effects, from asymptomatic infections in some individuals to fatal disease in others. Other researchers have reviewed mechanistic details regarding what is known about the virus. Here, the focus will be on the emerging data on host factors that might contribute to the wide range of severity.

There are many risk factors, along with extensive research related to each, so this discussion cannot be comprehensive. The focus of the first part of this review will be on the most common COVID-19 comorbidities[13] and what research suggests might be the safest and most easily altered lifestyle and dietary factors that might ameliorate them and possibly reduce COVID-19 morbidity and mortality.

\section{Aging-associated microbiota changes may be key to susceptibility rather than aging itself}

Age is clearly one of the primary factors correlated with severe outcomes of COVID-19 and may be related to immunosenescence-the gradual reduction in immune function typically observed with age[14]. However, chronological age alone may not be the most important factor in recovery from the viral infection, since people under 40 years old have died, while others, who are over 90 , have recovered. It is hypothesized here that factors that are often, but not always, related to aging are more important than age itself.

Clues as to these age-associated factors may come from the observation that individuals with the most severe outcomes frequently had preexisting conditions, including cardiovascular disease, hypertension, Type 2 diabetes mellitus and chronic lung diseases, such as chronic obstructive pulmonary disease (COPD) or asthma[1,3]. Thus, a brief examination of some of these diseases in relation to the microbiome and respiratory infections might be useful.

All these diseases are associated with inflammation and have been linked to various types of imbalances, or "dysbiosis," in the gut microbiota([15,16]. Microbes are also found in tissues in healthy controls, e.g., the blood[17] and lungs[18]. It has been hypothesized that what distinguishes the diseased state from a 
healthy state is increased levels of potentially pathogenic species from a variety of endogenous or exogenous sources[12].

Extensive evidence for the role of the microbiome comes from studies of the interactions between immune responses to various respiratory infections and the intestinal microbiome in animal models. A recent review[4] comprehensively surveys the research regarding a wide array of types of microbiome-viral interactions, noting that evidence supports the idea that a normal healthy microbiome tends to lead to resistance to viral infections. For example, in a chicken model of influenza, antibiotic-treated mice are more susceptible to influenza infection[19]. In a mouse model, the commensal bacteria, Clostridium orbiscendins, produces desaminotyrosine, which primes Type I interferon signaling, thus mediating protection from influenza[20]. In a model of respiratory syncytial virus, a probiotic was able to restore protection from viral infection in germ-free mice[21].

Many different microbes, including oral microbes associated with periodontal disease[22] and Chlamydia pneumoniae[23], have been found in atherosclerotic plaques, and these microbes have been proposed to lead to the chronic low-grade inflammation typical of atherosclerosis. COPD is associated with an abnormal or dysbiotic microbial community in the lungs[18] and the gut[24]. Diabetes and the obesity that is often associated with it are also characterized by inflammation and imbalanced microbial communities[25]. Although it is a low-grade inflammation, elevations of IL- 6 and CRP indicate that an inflammatory process is occurring[26]. Although these inflammatory markers tend to increase with age, along with rates of agingassociated diseases, they can be normal in those experiencing healthy aging[26] and can be high in younger people under certain circumstances[27].

Although not the topic of this article, it should be noted in passing that there are other factors that might influence susceptibility to COVID-19. A few of the possibilities include genetic effects on immune responses, nutritional status, prior chronic disease or therapy effects on immune responses and immune memory to coronaviruses or other potentially cross-reacting viruses. The infectious dose of the virus may also be important in determining outcomes.

\section{Air pollution effects on chronic disease and the potential role of the microbiome}

Another important factor influencing inflammation from cardiovascular disease, diabetes and chronic lung disease is air pollution. Increasing evidence is showing a quite substantial effect, even when the air pollution levels are below national standards[28-30], indicating a direct or indirect effect on lung pathology and quite possibly the microbiome. Although there are many potential effects of air pollution, this pattern is compatible with a role for the colonization/infection of microbes associated with air pollution, as described previously[12]. Little research has been done on this so far; however, changes in the nasopharyngeal microbiota were observed after a severe haze event in China[31]. It is also possible that hard-to-detect low-abundance microbes from the rare biosphere may also produce pro-inflammatory effects[12]. It has been observed that COPD exacerbations are often related to exposures to certain gases and particulate matter[32]. It seems possible that many of these gases and types of particulate matter, especially from the burning of fossil fuel, may have microbial strains associated with them that humans have not coevolved with. These relatively novel, potentially non-coevolved microbes (PHM) might be more prone to cause increased hypersensitivity reactions[12].

Not only are the above chronic diseases related to air pollution on a long-term basis, but increases in air pollution in the short-term lead to increases in emergency room visits, hospitalizations and deaths[33], including deaths from respiratory infections[34]. It is estimated that indoor and outdoor air pollution-related deaths annually exceed 7 million[35].

Recent studies in the U.S. and Italy also support a significant effect on mortality from COVID-19 from long-term air pollution exposure[36,37]. These potential COVID-19 mortality effects and the longer-term global effects mentioned above emphasize the need to reduce the use of polluting fossil fuels for health reasons in addition to avoiding the worst effects of global warming on many other facets of human health and well-being[38]. Smoking reduction is important, but it is also important to dramatically reduce and 
eventually eliminate unnecessary wood/biomass burning and fossil fuel-related pollution by adopting newer clean energy technologies.

\section{Diet, inflammation and the microbiome}

A "westernized" diet, including higher animal fat and animal products, increased sugar and ultra-processed foods and overconsumption of calorie-dense, low nutrient and low fiber foods appears to be an important factor in increasing disease rates in many countries around the world[39,40]. Allergic and autoimmune diseases have increased along with the adoption of this diet, but so have cardiovascular diseases, diabetes, obesity and chronic lung diseases[41,42]. This suggests that diet may be playing an important role in these diseases. This is supported by interventional studies in type 2 diabetes mellitus and cardiovascular disease $[43,44]$. Diet also appears to play a significant role in COPD[45].

Interleukin-6 (IL-6) and C-reactive protein (CRP) are correlated with obesity[46]. CRP has been found to decrease with weight loss independently of the type of diet or weight loss method[47]. Studies have found that the traditional Mediterranean diet, in particular, lowers IL-6, with part of its effect being independent of weight loss[48]. Dietary approaches to stop hypertension (DASH), emphasizes the consumption of low fat, whole foods. The DASH approach has been found to be effective in reversing cardiovascular disease and hypertension[44,49]. Another study showed that a diet with reduced saturated fat and increased fiber, combined with exercise, prevented diabetes in a proportion of patients in a randomized controlled trial of pre-diabetics[50]. Vegetarian or plant-based diets have shown benefit in cardiovascular disease[44] and other inflammatory diseases[51], including inflammatory arthritis[52]. Vegetarian and vegan diets appear to be generally safe, if vitamin B12 is supplemented and adequate attention is paid to other potentially deficient nutrients[53]. Diets that tend to emphasize fruits and vegetables and reduce processed meats and added sugar are associated with better outcomes with regard to lung function in COPD[45]. Typically, these diets cause changes in the microbiota that are beginning to be explored[54]. All tend to increase consumption of fiber, which many studies have found to affect short chain fatty acid production[55] and immune function[5].

Ruminococcus gnavus, a commensal microbe in the intestinal tract, was recently found to be potentially involved in contributing to inflammatory arthritis[56] and inflammatory bowel disease[57]. This may be due to several characteristics, including its ability to degrade mucus, which enhances its translocation across a weakened epithelial barrier. R. gnavus is also increased in those with coronary artery disease[58] and has been found to be decreased by a higher fiber, lower sugar Mediterranean diet[59]. A mutated clade of R. gnavus that tolerates a high-oxygen, pro-inflammatory environment in the intestinal tract has been identified in inflammatory bowel disease[60].

Enterococcus gallinarum, another commensal microbe with pathogenic potential, has been implicated in systemic lupus erythematosus[61]. E. gallinarum can cause disease in a lupus mouse model when the intestinal barrier is weakened, as can happen as a result of dietary factors, certain drugs, hyperglycemia and other factors[62].

There is evidence of a high-fat diet being associated with increases in endotoxin-producing, gram negative Enterobacteriaceae[63]. Endotoxin, as well as the bacteria that produce it, can cross the mucosal barrier of the gut and have systemic inflammatory effects. Lower levels of fiber are thought to increase mucin degrading bacteria that also increase intestinal permeability. Research indicates that this can negatively affect immune function and, therefore, promote respiratory diseases[63].

There is growing support for the view that many of the effects of diet on health arise from the effects of different types of food on the presence of pathogenic species or on the microbial balance in the intestines and elsewhere[12,62,64]. Thus, dietary patterns can lead to inflammation and the overabundance of inflammationassociated microbes, including Enterobacteriaceae or fungal species, such as Candida albicans, that have greater pathogenic potential. It has also been proposed that microbes in food/beverages may have harmful or beneficial effects, and microbes and other substances from ultra-processed foods may be more likely to have harmful effects[12]. 
The connection between the gut and lungs is increasingly apparent. One example is a study that found Candida albicans overgrowth in the intestinal tract had a negative effect on an inflammatory lung disease[65]. C. albicans may overgrow due to antibiotic use, excessive sugar and refined carbohydrates[66] or low-grade inflammation[67]. Other interactions between the gut and lung microbiotas have been discussed in recent reviews[4,68]

With regard to weight loss interventions, recent evidence from a randomized controlled study of inpatient adults indicates that increased consumption of ultra-processed foods is a key factor that leads to an overconsumption of carbohydrates resulting in weight gain[69]. Whether a diet that focuses on reducing carbohydrates or reducing fat is most beneficial, appears to vary between individuals as a result of still undetermined factors[70]. In fact, recent research using continuous blood sugar monitoring found that the precise foods that will increase blood sugar most, and thus be more likely to cause significant negative metabolic effects, differs among individuals[71,72]. Thus, the best approach to maintaining an optimal weight and blood sugar level may need to be personalized to some degree. However, a common theme in the diet research is the need to favor whole plant foods, particularly fruits and vegetables, and reduce ultra-processed food.

Recent research is showing the benefits of intermittent fasting, time-restricted eating[73] and fasting mimicking diets[74,75]. One benefit of a time-restricted approach appears to be related to it being typically more compatible with human circadian rhythms[76]. This approach may also facilitate reduction of overall caloric intake[73] and may improve sleep[76].

Recently, a carefully-designed diet that restricts overall calories and protein has been developed that is meant to be used for a period of 5 days every few months[77]. This fasting mimicking diet is designed to be easier to follow, yet have most of the benefits of fasting or caloric restriction. Studies suggest that it can increase stem cell production and the beneficial process of autophagy with overall beneficial effects on immune function[78]. There is also evidence that it is beneficial with regard to insulin resistance and diabetes[74] and has positive effects on the microbiota[79].

Besides these benefits and the benefit of weight loss programs in general, there may be other advantages of these types of dietary interventions. Food/beverages from a westernized diet, particularly high levels of high-fat, processed animal products and ultra-processed, high sugar/refined carbohydrate food, could cause imbalances in the microbiome and be a direct or indirect source of increased levels of pro-inflammatory, potentially pathogenic, microbes as discussed above[12]. Thus, dietary interventions that reduce these ultraprocessed foods/beverages may help give the immune system a better chance of reducing negative effects of these microbes.

Overall, the various dietary interventions discussed above appear to reduce the tendency of a westernized diet to contribute to insulin resistance, weight gain, microbial imbalances, infections and immune dysregulation. The above research does not determine whether a particular diet will be beneficial for avoiding severe disease from COVID-19, but it does suggest interventions that could help improve and, in some cases, prevent/reverse the diseases that are risk factors for it. Some suggestions for research specific to COVID-19 will be discussed near the end of this article.

\section{The role of psychological and physical stress in relation to the microbiome}

Chronic stress from psychological or physical factors has been shown to increase stress hormones, like cortisol, as well as inflammation and there is some evidence that mind-body interventions can be helpful in reversing these effects[80]. Chronic stress also can increase vulnerability to infection[81] and imbalances in the intestinal microbiome[82]. Greater psychological and physiological stress can result from many sources[12] and lead to reduction in secretory $\operatorname{IgA}[83]$, which is essential for protection from microbial invasion. A study in mice showed that lack of secretory IgA led to a COPD-like condition, as bacteria invaded the epithelial lining of the lung[84].

Stress reduction methods, such as diaphragmatic breathing, have shown benefits and a recent article[85] 
aimed at health care workers that must cope with intense psychological pressures in the current pandemic have suggested a number of "micropractices," including breathing practices. These methods could potentially help reduce stress even when minimal time is available. A recent review of diaphragmatic breathing (taking a deep breath, allowing the lung to expand toward a relaxed abdomen, holding it briefly and letting the breath out slowly) showed that such breathing practices reduced cortisol and resulted in other benefits as well[86].

The elevated cortisol associated with chronic stress and aging appears to be associated with inflammation[87] and has been linked to susceptibility to infection and difficulty recovering from stressors such as surgery[88]. Elevated cortisol is also associated with insulin resistance, which is linked to obesity, cardiovascular disease, hypertension and diabetes[89]. Interestingly, research is still clarifying the effects of cortisol on immune function. There is evidence that the effects are complex, with moderate levels of cortisol having delayed immune effects that can potentiate a pro-inflammatory response[90,91].

The hypothesis that stress, in some circumstances, may be part of a protective mechanism to cause avoidance of toxins and microbes that elicit allergic host defense responses has been discussed in detail elsewhere[12]. In 2 studies stress-related neuropsychiatric disorders have been associated with allergies $[92,93]$. In support of the causal role of allergy in this association, anxiety-like behavior has been observed in mice in response to small amounts of allergens in their cages, and this was found to be dependent on classical allergic mechanisms involving mast cells and $\operatorname{IgE}[94]$. Cortisol releasing factor (CRF) increases in the brain along with the anxiety-like behavior resulting from trace amounts of allergen in the cage was observed in similar rat model experiments[95]. Thus, the potential for physiological stress to result from allergy/hypersensitivity responses to both inhaled and ingested substances should be considered.

\section{Exercise, stress, immunity and the microbiome}

Over the last 100 years humans have tended to adopt a more sedentary lifestyle, and this appears to have been accelerated with westernization. Appropriate levels of exercise are associated with multiple benefits and appear to cause positive changes in the gut microbiome[96]. Even minimal amounts of exercise have been shown to be beneficial in helping maintain physical function and avoiding falls in the frail elderly[97]. Exercise has also been associated with reduced cardiovascular disease and all-cause mortality, indicating benefits even at only one third the recommended level[98,99].

However, excessive exercise, as in the overtraining syndrome, can increase stress and cause a decrease in sleep quality and immune function[100]. Thus, care must be taken to maintain an appropriate level of exercise for maximum health benefits. At least 150 minutes of moderate-intensity exercise per week has been recommended to be beneficial for the average person and even greater benefits were found at 3 to 5 times the recommended level[99]. However, the type and amount of exercise must be tailored to the health status of the individual.

Unfortunately, many people in westernized societies do not achieve the optimal level of exercise. There may be many reasons for this, including lack of energy and motivation. These problems might be ameliorated by a diet that is less associated with inflammation. Inflammation appears to have negative effects on brain areas associated with motivation[101] and has the potential to diminish energy production by mitochondria[102]. Neuroinflammation and impaired mitochondrial function have been linked to a highly processed high-fat diet fed to mice[103]. Thus, diets such as those discussed above that emphasize whole plant foods and appear to have anti-inflammatory effects may enhance the ability and motivation to engage in adequate exercise.

Thus, overall, there is evidence that air pollution and a westernized diet and lifestyle contribute to multiple common chronic diseases and their associated elevations in inflammation. It has been suggested that both air pollution and a westernized diet contribute to dysbiotic human microbiotas, both indirectly and directly, through post-hunter-gatherer era microbes and various substances present in polluted air and a westernized diet[12]. It might be postulated that the immune reactions to these pathogenic/dysbiotic microbes may be kept to a relatively low-grade inflammation because immune system signaling only indicates the presence 
of a slow-growing chronic microbial colonization/infection rather than a rapidly increasing microbial threat. However, this immune system signaling could change in the context of an acute infection, as discussed further below.

\section{Chronic low-grade inflammation transformed to acute life-threatening inflamma- tion?}

In COVID-19, a severe cytokine storm can occur with high levels of IL-6, which often leads to acute respiratory distress syndrome (ARDS), organ failure and death[3]. It is intriguing to consider that this involvement of high IL-6 and other inflammatory markers might suggest that the excessive immune response of the cytokine storm that occurs in severe COVID-19 might be at least partly related to the elevated inflammatory markers in the comorbid conditions discussed above.

It is interesting to consider the hypothesis mentioned above that abnormal microbiota are causing low-grade infection or dysbiosis, and chronically stimulating an immune response against them and, consequently, elevating IL-6 and CRP. Then, when the acute increase in the innate immune response occurs during the COVID-19 infection, the immune system reacts against the dysbiotic microbes as well as SARS-CoV-2. And the overall immune activation leads to antibodies against the coronavirus, but also higher levels of antibodies to some of the microbes of the dysbiotic microbiome.

Thus, the immune response targeting at least some microbial strains in the microbiome might be at least part of what leads to the excessive, dysregulated immune response in those with severe COVID-19. At least some of these dysbiotic microbes are likely to not be amenable to being eliminated by the immune system for various reasons discussed elsewhere, such as heterologous infection and immunodominance[12]. Thus, the increased immune response against these dysbiotic microbes during COVID-19 may not always be reversible and instead may lead to prolonged intense inflammation culminating in organ failure.

Severe COVID-19 disease in younger people could be at least partly explained by dysbiotic microbiomes occurring earlier than usual due to a variety of chemical and microbial exposures, inherited microbiomes, air pollution, occupational exposures, diet and lifestyle factors, as well as previous antibiotic use, which might also lead to elevated IL-6 and CRP. The ability of some healthy elderly patients to recover from infection with COVID-19 could be due to a more balanced or "healthy" microbiome with little, if any, of the more pathogenic/dysbiotic species or strains, as has been indicated in association with healthy aging[104].

Other factors are also likely to be involved; however, it is possible that the microbiome present prior to the viral infection could play an important role in the outcome of COVID-19.

\section{Some possible implications of microbiome and immune system inter- actions for treatment of severe COVID-19}

The drugs that are currently being tested to treat severe COVID-19 infection are primarily focused on preventing or slowing viral replication or blocking the actions of IL-6 or other cytokines. In order to consider a wide range of possibilities to help achieve the greatest potential for benefit, it seems worth considering the role of other immune cells that contribute to the production of IL- 6 and other inflammation-related changes.

As mentioned previously, the tendency for severe COVID-19 to be most common in individuals with chronic diseases that may involve infections/dysbiosis that produce low-grade inflammation would suggest that other approaches might also be beneficial. Previously, the growing evidence for a significant role for mast cells in a number of chronic conditions that involve elevated pro-inflammatory cytokines and hypersensitivity responses was discussed[12]. Mast cells also appear to play an important role in cardiovascular disease[105], diabetes[106] and COPD[107]. The many effects of mast cells on inflammatory processes in cardiovascular 
disease, obesity, insulin resistance and diabetes, as well as established and experimental medications to prevent or block these effects, was reviewed by Theoharides et al[108].

Hypertension, associated with elevated angiotensin II, has been linked to mast cells[109], and mast cell stabilization has lowered angiotensin II in animal models[110]. It has been suggested that an approach to treating hypertension that is focused on mast cells might be worth studying in humans[110]. Activation of the renin angiotensin aldosterone system (RAAS) has been found to occur as a result of acute psychosocial stress[111] and may also occur as a result of chronic stress[112]. Elevated angiotensin II appears to be associated with more severe COVID-19[113]. The elevation is thought to be due to the actions of the coronavirus binding ACE2 in a way that reduces its ability to degrade angiotensin II[114]. The fact that hypertension seems to be found at a higher level among the most severe COVID-19 cases, suggests that processes that were occurring before the viral infection might be relevant as well. It is interesting to consider the possibility that a prior elevation of angiotensin II in hypertensive patients might play a role in the severity of COVID-19. Research has shown that inflammation is interconnected extensively with activation of the RAAS[115]. It seems reasonable to speculate that the prior inflammation and stress associated with an increased activation of the RAAS might arise from low grade infections and/or dysbiotic microbiotas, as discussed above[114], and might be a contributing factor leading to the development of severe COVID-19.

Mast cell stabilization has shown benefits in a randomized controlled trial of diabetes in humans[116] and is discussed in a recent review on cardiovascular disease[105]. Additional research on the role of IgE and mast cells has been reviewed[106,117], indicating a potential beneficial role of mast cell stabilization in obesity and diabetes as well.

Besides their role in allergies/hypersensitivities, mast cells are important in the immune response to viral, bacterial, fungal and parasitic infections[118] and there is evidence that they play a role in the immunopathology in coronavirus infections[119,120]. Mast cells are important in immune defense; however, when mast cell activation is excessive, it can cause more harm than benefit. Studies in mice support the role of excessive activation of mast cells in viral respiratory illnesses, such as influenza, that cause death largely due to excessive inflammation[121]. Sodium cromoglycate, which stabilizes mast cells, has been shown to be beneficial in a mouse model of H5N1 influenza[122]. Mast cell stabilization using ketotifen reduced lung lesions and apoptosis in another study using a mouse model of H5N1 influenza[123]. Kritas et al[119] has suggested that a possible treatment approach to control the mast cell contribution to the high level of inflammation in severe COVID-19 might involve anti-inflammatory cytokines of the IL-1 family.

Mast cell activation appears to be important in lung diseases[107], like COPD, one of the diseases that is a risk factor for severe COVID-19 outcomes. Fibroproliferation that occurs in the acute respiratory distress syndrome may not be initiated by mast cells; however, mast cells still appear to play an important role in the increased inflammation[124]. Overed-Sayer[125] et al reviewed studies on the mast cell role in idiopathic pulmonary fibrosis and fibrotic diseases of other organs, indicating that the role of mast cells is a promising area of investigation.

Sepsis occurs in a subset of severe COVID-19 cases[126]. In animal models, sepsis has been linked to mast cell involvement[127] and has been improved by mast cell stabilization[128] and histamine receptor blockers[129]. Mast cell activation seems to be beneficial at the local level or more moderate disease, but was detrimental at the systemic level in the severe cecal ligation and puncture (CLP) mouse model of sepsis[118,130,131]. On the whole, these and other studies and reviews suggest that mast cell focused approaches are worth investigating as treatment approaches in COVID-19.

COPD is a condition associated with severe outcomes of COVID-19 and may be linked to other risk factors for severe COVID-19, such as diabetes[132] and heart disease[133]. It is estimated that an increasing portion of the U.S. population has reduced airway function related to COPD and that $70 \%$ of these cases remain undiagnosed[134,135]. Many cases are related to tobacco use; however, research[136] has also implicated other factors, such as occupational and other environmental exposures, such as air pollution and mold exposure[137]. 
It is also interesting to consider a potential role for hypersensitivity-related mechanisms and treatments. Asthma is often associated with reactions to allergens or chemicals/irritants, and asthma can be difficult to distinguish from COPD, especially in the elderly[138]. It has even been proposed that asthma and COPD may be part of the same disease process, particularly because prior asthma is associated with the development of COPD[139]. Asthma-COPD overlap syndrome is increasingly being recognized and is found to benefit from the IgE-blocking monoclonal antibody, omaluzimab[139]. Atopy has been found at a significant level in dairy farmers with COPD[140].

Both allergic and non-allergic rhinitis are common comorbid conditions in COPD, and even though IgE may not be elevated in the serum in many rhinitis patients, non-allergic rhinitis has been associated with local elevations of $\operatorname{IgE}[141]$. IgE levels are thought to decline to some degree with age, like other immunoglobulins, as part of immunosenescence. However, allergic diseases can still be significant in the elderly, despite the fact that they are often given less attention[142].

It is interesting to consider whether IgE responses might, under certain circumstances, shift over time, toward excessive IgG responses that have the potential for pathogenic effects, as has been suggested in eosinophilic esophagitis[143] and possibly other conditions[12]. Both IgE and IgG responses might be targeting at least some microbes within the tissue microbiota and possibly cross-reacting self-antigens. Trost et al has found cross-reactivity between microbes and tissue antigens to be extensive[144].

In severe viral infections, such as COVID-19, the activation of the immune system in response to the coronavirus might cause an increased immune response to the dysbiotic microbiota and cross-reacting self-tissues and could be hypothesized to be a factor in the development of the acute respiratory distress syndrome and multiple organ failure in COVID-19.

Based on the above connections between COPD and allergic conditions, IgE levels might be measured in COVID-19 patients, especially in those with prior lung disease, like asthma or COPD. Typically, IgE levels are high at the beginning of a viral respiratory infection and they decline over a 3-month period. But perhaps, they would stay high in more severely ill COVID-19 patients. This pattern was found in atopic individuals with viral infections[145]. If this were the case, or if local IgE levels were elevated, the IgEtargeting monoclonal antibody drug, omaluzimab, might be considered in some patients as a treatment for severe COVID-19 inflammation. Omaluzimab is thought to work through stabilizing mast cells as well as binding IgE. The large numbers of those with undiagnosed COPD mentioned above might be reason to consider this approach even in those without known prior chronic lung disease.

Other inflammatory markers are also being implicated in severe COVID-19 and a detailed discussion of this topic is beyond the scope of this review. However, among 3 markers that Yang et al[146] found were associated with worse outcomes of COVID-19 was IP-10 (aka interferon gamma-induced protein-10 or CXCL-10 or C$\mathrm{X}-\mathrm{C}$ motif chemokine 10). They suggested IP-10 might be a therapeutic target to consider in the treatment of COVID-19. This marker has also been associated with cardiovascular disease and risk of diabetes[147] as well as the recruitment of mast cells in asthma[148]. Evidence suggests that IP-10 also plays a role in $\operatorname{COPD}[149]$.

Another consideration relevant to a role for the microbiome or other infectious agents in COVID-19 is the possibility of a bacterial or fungal strain being present in the alveoli of some individuals that might crossreact with SARS-CoV-2 and thus might contribute to inflammatory symptoms (L. Carrasco, UAM, personal communication). Interestingly, hydroxychloroquine has been found to have antifungal and antibacterial effects. For instance, there was a case report of a skin infection with Aspergillus niger resolving in the weeks after hydroxychloroquine was instituted for a rheumatic condition only to return when the patient had to stop the hydroxychloroquine due to tinnitus[150]. There have been promising reports regarding the use of hydroxychloroquine in SARS-CoV-2 with a focus on its antiviral effects[151]. Other recent studies have failed to show benefit $[152,153]$, however, further large randomized controlled trials are being conducted. If shown to be effective in at least a proportion of patients, it might be acting via multiple antimicrobial mechanisms. It might be that hydroxychloroquine or another antimicrobial would be most beneficial if administered before 
the severe inflammatory stage that requires anti-inflammatory medications. Since antimicrobials also may have harmful effects, including disruptive effects on the microbiome, caution needs to be used. Microbiome studies would be useful to test this hypothesis.

\section{Suggested future research}

Based on the above considerations, it is suggested that studies should be conducted to assess the dietary patterns and other lifestyle factors of people who have asymptomatic, mild or severe COVID-19 infections to determine what factors are most associated with various outcomes. During the pandemic crisis, it may be difficult to do these types of studies, but it might be possible via telephone or online surveys of families of those who are ill and/or hospitalized, to assess eating patterns and lifestyle factors of those who got sick and those who did not. For those who are deceased, information on dietary patterns and lifestyle factors could be obtained from close relatives.

Eventually, when there is improved availability of testing and more types of tests for COVID-19, including tests for past exposure using antibody measurements, it should become easier to do these types of studies. Of course, prospective studies are preferable, however, they may not be possible in many cases in the midst of the current pandemic.

Preferably, samples of the microbiome from a variety of sites, perhaps including oral, fecal, blood, urinary and respiratory tract, would be gathered before the illness develops to help assess microbiome-related risk factors. If this is not possible, information on the microbiome might be inferred from diet and lifestyle data, drawing on the growing amount of research on the relationship between these factors and the microbiome composition. The use of large databases of previously gathered microbiome data might also be considered for inclusion in these types of studies. In some cases, autopsy samples of many different organs could be analyzed to attempt to detect microbiome-related patterns.

In addition, the expansion of the types of immune system manipulation methods being studied in cases of severe COVID-19 might be considered. As mentioned above, research could be conducted on medications that are related to mast cell function and/or inhibit hypersensitivity reactions. The use of microbiota manipulation or antimicrobials that target components of a potentially dysbiotic microbiota could be considered. These might be studied alone or in combination with other treatment approaches.

\section{Conclusions}

The age-associated diseases that appear to be most associated with severe COVID-19 are also associated with elevated IL-6 and other inflammatory markers, along with evidence of microbiome dysbiosis and/or low-grade infection. The imbalances in the immune system arising from this potentially microbiome-related inflammation are possibly a contributing factor in the susceptibility to acute viral infection and the severe cytokine storms that frequently lead to fatal outcomes in COVID-19.

In the long run, determination of how to achieve an optimal response to viral infections like COVID-19 will likely benefit from increased studies on how the microbiome might be shifted toward a more normal, healthy, youthful state. Based on the research discussed above, this state would be expected to be associated with a lower likelihood of severe effects from COVID-19 and other infectious agents, along with benefit in other debilitating diseases. It would be preferable to achieve this state with lifestyle and dietary factors; however, medications, probiotics and other approaches discussed in this review could also be used if supported by adequate research. To achieve this goal and the goal of improving COVID-19 outcomes, more research is clearly needed.

\section{Funding}


This review was done without external funding.

\section{Acknowledgements}

I gratefully acknowledge Ian Roberts-Thomson, M.D., for helpful comments that helped improve the manuscript. I thank David Waterhouse, Ph.D., for editorial assistance.

\section{Competing interests}

JCW has plans to file one or more patents that are potentially related to some of the ideas included in this review and plans to donate all proceeds from her share of any profits from them to charity, including charities that fund medical research.

\section{References}

[1] Ruan Q, Yang K, Wang W, Jiang L, Song J. Clinical predictors of mortality due to COVID-19 based on an analysis of data of 150 patients from Wuhan, China. Intensive Care Med 2020:1-3. doi:10.1007/s00134020-05991-x.

[2] Cao B, Wang Y, Wen D, Liu W, Wang J, Fan G, et al. A Trial of Lopinavir-Ritonavir in Adults Hospitalized with Severe Covid-19. N Engl J Med 2020:NEJMoa2001282. doi:10.1056/NEJMoa2001282.

[3] Zhou F, Yu T, Du R, Fan G, Liu Y, Liu Z, et al. Clinical course and risk factors for mortality of adult inpatients with COVID-19 in Wuhan, China: a retrospective cohort study. Lancet 2020;395:1054-62. doi:10.1016/S0140-6736(20)30566-3.

[4] Li N, Ma W-T, Pang M, Fan Q-L, Hua J-L. The Commensal Microbiota and Viral Infection: A Comprehensive Review. Front Immunol 2019;10:1551. doi:10.3389/fimmu.2019.01551.

[5] Dang AT, Marsland BJ. Microbes, metabolites, and the gut-lung axis. Mucosal Immunol 2019;12:843-50. doi:10.1038/s41385-019-0160-6.

[6] Sender R, Fuchs S, Milo R. Are We Really Vastly Outnumbered? Revisiting the Ratio of Bacterial to Host Cells in Humans. Cell 2016;164:337-40. doi:10.1016/j.cell.2016.01.013.

[7] Brown JM, Hazen SL. Targeting of microbe-derived metabolites to improve human health: The next frontier for drug discovery. J Biol Chem 2017:8560-8. doi:10.1074/jbc.R116.765388.

[8] Grice EA, Segre JA. The Human Microbiome: Our Second Genome. Annu Rev Genomics Hum Genet 2012;13:151-70. doi:10.1146/annurev-genom-090711-163814.

[9] Shogbesan O, Poudel DR, Victor S, Jehangir A, Fadahunsi O, Shogbesan G, et al. A Systematic Review of the Efficacy and Safety of Fecal Microbiota Transplant for Clostridium difficile Infection in Immunocompromised Patients. Can J Gastroenterol Hepatol 2018;2018:1394379. doi:10.1155/2018/1394379.

[10] Strachan DP. Hay fever, hygiene, and household size. BMJ 1989;299:1259-60. doi:10.1136/bmj.299.6710.1259.

[11] Shreiner A, Huffnagle GB, Noverr MC. The "Microflora Hypothesis" of Allergic Disease. Adv Exp Med Biol 2008;635:113-34.

[12] Waterhouse J. Post-hunter-gatherer era microbes' role in allergic/autoimmune disease. Authorea Prepr 2020. doi:10.22541/AU.158035512.24828861.

[13] Preliminary Estimates of the Prevalence of Selected Underlying Health Conditions Among Patients with Coronavirus Disease 2019 - United States, February 12-March 28, 2020. MMWR Morb Mortal Wkly Rep 2020;69:382-386. doi:10.15585/mmwr.mm6913e2external icon. 
[14] Fulop T, Larbi A, Dupuis G, Le Page A, Frost EH, Cohen AA, et al. Immunosenescence and InflammAging As Two Sides of the Same Coin: Friends or Foes? Front Immunol 2018;8:1960. doi:10.3389/fimmu.2017.01960.

[15] Du Y, Li X, Su C, Wang L, Jiang J, Hong B. The human gut microbiome - a new and exciting avenue in cardiovascular drug discovery. Expert Opin Drug Discov 2019;14:1037-52. doi:10.1080/17460441.2019.1638909.

[16] Vaughan A, Frazer ZA, Hansbro PM, Yang IA. COPD and the gut-lung axis: the therapeutic potential of fibre. J Thorac Dis 2019;11:S2173-80. doi:10.21037/jtd.2019.10.40.

[17] Potgieter M, Bester J, Kell DB, Pretorius E. The dormant blood microbiome in chronic, inflammatory diseases. FEMS Microbiol Rev 2015;39:567-91. doi:10.1093/femsre/fuv013.

[18] Dickson RP, Martinez FJ, Huffnagle GB. The Role of the Microbiome in Exacerbations of Chronic Lung Diseases. Lancet 2014;384:691-702. doi:10.1016/s0140-6736(14)61136-3.

[19] Yitbarek A, Taha-Abdelaziz K, Hodgins DC, Read L, Nagy É, Weese JS, et al. Gut microbiota-mediated protection against influenza virus subtype H9N2 in chickens is associated with modulation of the innate responses. Sci Rep 2018;8:1-12. doi:10.1038/s41598-018-31613-0.

[20] Steed AL, Christophi GP, Kaiko GE, Sun L, Goodwin VM, Jain U, et al. The microbial metabolite desaminotyrosine protects from influenza through type I interferon. Science (80- ) 2017;357:498-502. doi:10.1126/science.aam5336.

[21] Ji J, Sun Q, Wang Q, Zhang H, Qin F, Wang Q, et al. Probiotics Confers Protection Against RSV Infections by Regulating Gut and Lung Microbiotas to Activate Antiviral Responses of Alveolar Macrophage. SSRN Prepr 2019. doi:10.2139/ssrn.3471990.

[22] Liu X-R, Xu Q, Xiao J, Deng Y-M, Tang Z-H, Tang Y-L, et al. Role of oral microbiota in atherosclerosis. Clin Chim Acta 2020;506:191-5. doi:10.1016/j.cca.2020.03.033.

[23] Joshi R, Khandelwal B, Joshi D, Gupta OP. Chlamydophila Pneumoniae Infection and Cardiovascular Disease. N Am J Med Sci 2013;5:169-81. doi:10.4103/1947-2714.109178.

[24] Zhang D, Li S, Wang N, Tan H-Y, Zhang Z, Feng Y. The Cross-Talk Between Gut Microbiota and Lungs in Common Lung Diseases. Front Microbiol 2020;11:301. doi:10.3389/fmicb.2020.00301.

[25] Adeshirlarijaney A, Gewirtz AT. Considering gut microbiota in treatment of type 2 diabetes mellitus. Gut Microbes 2020;2020:1-12. doi:10.1080/19490976.2020.1717719.

[26] Arai Y, Martin-Ruiz CM, Takayama M, Abe Y, Takebayashi T, Koyasu S, et al. Inflammation, But Not Telomere Length, Predicts Successful Ageing at Extreme Old Age: A Longitudinal Study of Semisupercentenarians. EBioMedicine 2015;2:1549-58. doi:10.1016/j.ebiom.2015.07.029.

[27] Beharka AA, Meydani M, Wu D, Leka LS, Meydani A, Meydani SN. Interleukin-6 Production Does Not Increase With Age. J Gerontol A Biol Sci Med Sci 2001;56:B81-8. doi:10.1093/gerona/56.2.B81.

[28] Ward-Caviness CK, Weaver AM, Buranosky M, Pfaff ER, Neas LM, Devlin RB, et al. Associations Between Long-Term Fine Particulate Matter Exposure and Mortality in Heart Failure Patients. J Am Hear Assoc 2020;9:e012517. doi:10.1161/JAHA.119.012517.

[29] Shah AS, Langrish JP, Nair H, McAllister DA, Hunter AL, Donaldson K, et al. Global association of air pollution and heart failure: a systematic review and meta-analysis. Lancet 2013;382:1039-48. doi:10.1016/S0140-6736(13)60898-3.

[30] Requia WJ, Adams MD, Arain A, Papatheodorou S, Koutrakis P, Mahmoud M. Global Association of Air Pollution and Cardiorespiratory Diseases: A Systematic Review, Meta-Analysis, and Investigation of Modifier Variables. Am J Public Health 2018;108:S123-s130. doi:10.2105/AJPH.2017.303839. 
[31] Qin T, Zhang F, Zhou H, Ren H, Du Y, Liang S, et al. High-Level PM2.5/PM10 Exposure Is Associated With Alterations in the Human Pharyngeal Microbiota Composition. Front Microbiol 2019;10:54. doi:10.3389/fmicb.2019.00054.

[32] Su Y-C, Jalalvand F, Thegerström J, Riesbeck K. The Interplay Between Immune Response and Bacterial Infection in COPD: Focus Upon Non-typeable Haemophilus influenzae. Front Immunol 2018;9:2530. doi:10.3389/fimmu.2018.02530.

[33] Samek L. Overall human mortality and morbidity due to exposure to air pollution. Int J Occup Med Environ Health 2016;29:417-26. doi:10.13075/ijomeh.1896.00560.

[34] Croft DP, Zhang W, Lin S, Thurston SW, Hopke PK, Masiol M, et al. The Association between Respiratory Infection and Air Pollution in the Setting of Air Quality Policy and Economic Change. Ann Am Thorac Soc 2019;16:321-30. doi:10.1513/AnnalsATS.201810-691OC.

[35] Babatola SS. Global burden of diseases attributable to air pollution. J Public Health Africa 2018;9:813. doi:10.4081/jphia.2018.813.

[36] Wu X, Nethery RC, Sabath BM, Braun D, Dominici F. Exposure to air pollution and COVID-19 mortality in the United States. MedRxiv Prepr 2020. doi:10.1101/2020.04.05.20054502.

[37] Conticini E, Frediani B, Caro D. Can atmospheric pollution be considered a co-factor in extremely high level of SARS-CoV-2 lethality in Northern Italy? Environ Pollut 2020;2020:114465. doi:10.1016/j.envpol.2020.114465.

[38] Rossati A. Global Warming and Its Health Impact. Int J Occup Environ Med 2017;8:7-20. doi:10.15171/ijoem.2017.963.

[39] Zinöcker MK, Lindseth IA. The Western Diet-Microbiome-Host Interaction and Its Role in Metabolic Disease. Nutrients 2018;10:365. doi:10.3390/nu10030365.

[40] Kopp W. How Western Diet And Lifestyle Drive The Pandemic Of Obesity And Civilization Diseases. Diabetes Metab Syndr Obes 2019;12:2221-36. doi:10.2147/DMSO.S216791.

[41] Keller KB, Lemberg L. Obesity and the metabolic syndrome. Am J Crit Care 2003;12:167-70.

[42] Guilleminault L, Williams EJ, Scott HA, Berthon BS, Jensen M, Wood LG. Diet and Asthma: Is It Time to Adapt Our Message? Nutrients 2017;9:1227. doi:10.3390/nu9111227.

[43] Hallberg SJ, Gershuni VM, Hazbun TL, Athinarayanan SJ. Reversing Type 2 Diabetes: A Narrative Review of the Evidence. Nutrients 2019;11:766. doi:10.3390/nu11040766.

[44] Ornish D, Brown SE, Scherwitz LW, Billings JH, Armstrong WT, Ports TA, et al. Can lifestyle changes reverse coronary heart disease? The Lifestyle Heart Trial. Lancet 1990;336:129-33. doi:10.1016/01406736(90)91656-u.

[45] Scoditti E, Massaro M, Garbarino S, Toraldo DM. Role of Diet in Chronic Obstructive Pulmonary Disease Prevention and Treatment. Nutrients 2019;11:1357. doi:10.3390/nu11061357.

[46] Khaodhiar L, Ling P, Blackburn G, Bistrian B. Serum Levels of interleukin-6 and C-reactive Protein Correlate With Body Mass Index Across the Broad Range of Obesity. JPEN J Parenter Enteral Nutr 2004;28:410-5. doi:10.1177/0148607104028006410.

[47] Selvin E, Paynter NP, Erlinger TP. The Effect of Weight Loss on C-Reactive Protein: A Systematic Review. Arch Intern Med 2007;167:31-9. doi:10.1001/archinte.167.1.31.

[48] Smidowicz A, Regula J. Effect of Nutritional Status and Dietary Patterns on Human Serum C-Reactive Protein and Interleukin-6 Concentrations. Adv Nutr 2015;6:738-47. doi:10.3945/an.115.009415.

[49] Ornish D. Avoiding revascularization with lifestyle changes: The Multicenter Lifestyle Demonstration Project. Am J Cardiol 1998;82:72T-76T. doi:10.1016/s0002-9149(98)00744-9. 
[50] Tuomilehto J, Lindström J, Eriksson JG, Valle TT, Hämäläinen H, Ilanne-Parikka P, et al. Prevention of Type 2 Diabetes Mellitus by Changes in Lifestyle among Subjects with Impaired Glucose Tolerance. N Engl J Med 2001;344:1343-50. doi:10.1056/NEJM200105033441801.

[51] Chiba M, Ishii H, Komatsu M. Recommendation of plant-based diets for inflammatory bowel disease. Transl Pediatr 2019;8:23-7. doi:10.21037/tp.2018.12.02.

[52] Jethwa H, Prince M, Bukhari M, Abraham S. The evidence for dietary manipulation in inflammatory arthritis. Int J Clin Rheumtol 2019;14:190-9.

[53] Sebastiani G, Barbero AH, Borrás-Novell C, Casanova MA, Aldecoa-Bilbao V, Andreu-Fernández V, et al. The Effects of Vegetarian and Vegan Diet during Pregnancy on the Health of Mothers and Offspring. Nutrients 2019;11:557. doi:10.3390/nu11030557.

[54] De Angelis M, Ferrocino I, Calabrese FM, De Filippis F, Cavallo N, Siragusa S, et al. Diet influences the functions of the human intestinal microbiome. Sci Rep 2020;10:4247. doi:10.1038/s41598-020-61192-y.

[55] Morrison KE, Jašarević E, Howard CD, Bale TL. It's the fiber, not the fat: significant effects of dietary challenge on the gut microbiome. Microbiome 2020;8:15. doi:10.1186/s40168-020-0791-6.

[56] Vereecke L, Elewaut D. Spondyloarthropathies: Ruminococcus on the horizon in arthritic disease. Nat Rev Rheumatol 2017;13:574-6. doi:10.1038/nrrheum.2017.130.

[57] Schirmer M, Garner A, Vlamakis H, Xavier RJ. Microbial genes and pathways in inflammatory bowel disease. Nat Rev Microbiol 2019;17:497-511. doi:10.1038/s41579-019-0213-6.

[58] Toya T, Corban MT, Marrietta E, Horwath IE, Lerman LO, Murray JA, et al. Coronary artery disease is associated with an altered gut microbiome composition. PLoS One 2020;15:e0227147. doi:10.1371/journal.pone.0227147.

[59] Meslier V, Laiola M, Roager HM, De Filippis F, Roume H, Quinquis B, et al. Mediterranean diet intervention in overweight and obese subjects lowers plasma cholesterol and causes changes in the gut microbiome and metabolome independently of energy intake. Gut 2020;2020:gutjnl-2019-320438. doi:10.1136/gutjnl2019-320438.

[60] Hall AB, Yassour M, Sauk J, Garner A, Jiang X, Arthur T, et al. A novel Ruminococcus gnavus clade enriched in inflammatory bowel disease patients. Genome Med 2017;9:103. doi:10.1186/s13073-017-0490-5.

[61] Vieira SM, Hiltensperger M, Kumar V, Zegarra-Ruiz D, Dehner C, Khan N, et al. Translocation of a gut pathobiont drives autoimmunity in mice and humans. Science 2018;359:1156-61. doi:10.1126/science.aar7201.

[62] Fine RL, Manfredo Vieira S, Gilmore MS, Kriegel MA. Mechanisms and consequences of gut commensal translocation in chronic diseases. Gut Microbes 2020;11:217-30. doi:10.1080/19490976.2019.1629236.

[63] Wypych TP, Marsland BJ, Ubags NDJ. The Impact of Diet on Immunity and Respiratory Diseases. Ann Am Thorac Soc 2017;14(S_5):S339-47. doi:10.1513/AnnalsATS.201703-255AW.

[64] Bailey MA, Holscher HD. Microbiome-Mediated Effects of the Mediterranean Diet on Inflammation. Adv Nutr 2018;9:193-206. doi:10.1093/advances/nmy013.

[65] Bacher P, Hohnstein T, Beerbaum E, Röcker M, Blango MG, Kaufmann S, et al. Human Anti-fungal Th17 Immunity and Pathology Rely on Cross-Reactivity against Candida albicans. Cell 2019;176:13401355.e15. doi:10.1016/j.cell.2019.01.041.

[66] Hills RD, Jr., Pontefract BA, Mishcon HR, Black CA, Sutton SC, et al. Gut Microbiome: Profound Implications for Diet and Disease. Nutrients 2019;11:1613. doi:10.3390/nu11071613.

[67] Kumamoto CA. Inflammation and gastrointestinal Candida colonization. Curr Opin Microbiol 2011;14:386391. doi:10.1016/j.mib.2011.07.015. 
[68] Invernizzi R, Lloyd CM, Molyneaux PL. Respiratory microbiome and epithelial interactions shape immunity in the lungs. Immunology 2020:10.1111/imm.13195. doi:10.1111/IMM.13195.

[69] Hall KD, Ayuketah A, Brychta R, Cai H, Cassimatis T, Chen KY, et al. Ultra-Processed Diets Cause Excess Calorie Intake and Weight Gain: An Inpatient Randomized Controlled Trial of Ad Libitum Food Intake. Cell Metab 2019;30:67-77.e3. doi:10.1016/j.cmet.2019.05.008.

[70] Gardner CD, Trepanowski JF, Del Gobbo LC, Hauser ME, Rigdon J, Ioannidis JPA, et al. Effect of LowFat vs Low-Carbohydrate Diet on 12-Month Weight Loss in Overweight Adults and the Association With Genotype Pattern or Insulin Secretion: The DIETFITS Randomized Clinical Trial. JAMA 2018;319:667-79. doi:10.1001/jama.2018.0245.

[71] Zeevi D, Korem T, Zmora N, Israeli D, Rothschild D, Weinberger A, et al. Personalized Nutrition by Prediction of Glycemic Responses. Cell 2015;163:1079-94. doi:10.1016/j.cell.2015.11.001.

[72] Elinav E, Segal E, Adamson E. The personalized diet : the pioneering program to lose weight and prevent disease. 1st ed. NY, NY: Grand Central Publishing,; 2017.

[73] Lee SA, Sypniewski C, Bensadon BA, McLaren C, Donahoo WT, Sibille KT, et al. Determinants of Adherence in Time-Restricted Feeding in Older Adults: Lessons from a Pilot Study. Nutrients 2020;12:E874. doi:10.3390/nu12030874.

[74] Wei S, Zhao J, Bai M, Li C, Zhang L, Chen Y. Comparison of glycemic improvement between intermittent calorie restriction and continuous calorie restriction in diabetic mice. Nutr Metab (Lond) 2019;16:60. doi:10.1186/s12986-019-0388-x.

[75] Longo VD, Juventology S. Programmed longevity, youthspan, and juventology. Aging Cell 2019;18:e12843. doi:10.1111/acel.12843.

[76] Longo VD, Panda S. Fasting, circadian rhythms, and time restricted feeding in healthy lifespan. Cell Metab 2016;23:1048-59. doi:10.1016/j.cmet.2016.06.001.

[77] Longo V. Longevity Diet : slow aging, fight disease, optimize weight. NY, NY: Avery Pub Group; 2019.

[78] Cheng C-W, Villani V, Buono R, Wei M, Kumar S, Yilmaz OH, et al. Fasting-mimicking diet promotes Ngn3-driven $\beta$-cell regeneration to reverse diabetes. Cell 2017;168:775-788.e12. doi:10.1016/j.cell.2017.01.040.

[79] Rangan P, Choi I, Wei M, Navarrete G, Guen E, Brandhorst S, et al. Fasting-Mimicking Diet Modulates Microbiota and Promotes Intestinal Regeneration to Reduce Inflammatory Bowel Disease Pathology. Cell Rep 2019;26:2704-2719.e6. doi:10.1016/j.celrep.2019.02.019.

[80] Buric I, Farias M, Jong J, Mee C, Brazil IA. What Is the Molecular Signature of Mind-Body Interventions? A Systematic Review of Gene Expression Changes Induced by Meditation and Related Practices. Front Immunol 2017;8:670. doi:10.3389/FIMMU.2017.00670.

[81] Pedersen A, Zachariae R, Bovbjerg DH. Influence of Psychological Stress on Upper Respiratory InfectionA Meta-Analysis of Prospective Studies. Psychosom Med 2010;72:823-32. doi:10.1097/PSY.0b013e3181f1d003.

[82] Rea K, Dinan TG, Cryan JF. The microbiome: A key regulator of stress and neuroinflammation. Neurobiol Stress 2016;4:23-33. doi:10.1016/j.ynstr.2016.03.001.

[83] Dragoş D, Tănăsescu M. The effect of stress on the defense systems. J Med Life 2010;3:10-8.

[84] Richmond BW, Brucker RM, Han W, Du R-H, Zhang Y, Cheng D-S, et al. Airway bacteria drive a progressive COPD-like phenotype in mice with polymeric immunoglobulin receptor deficiency. Nat Commun 2016;7:11240. doi:10.1038/ncomms11240.

[85] Fessell D, Cherniss C. Coronavirus Disease 2019 (COVID-19) and Beyond: Micropractices for Burnout Prevention and Emotional Wellness. J Am Coll Radiol 2020;S1545-1440. doi:10.1016/j.jacr.2020.03.013. 
[86] Ma X, Yue Z-Q, Gong Z-Q, Zhang H, Duan N-Y, Shi Y-T, et al. The Effect of Diaphragmatic Breathing on Attention, Negative Affect and Stress in Healthy Adults. Front Psychol 2017;8:874. doi:10.3389/fpsyg.2017.00874.

[87] Lavretsky H, Newhouse PA. Stress, Inflammation and Aging. Am J Geriatr Psychiatry 2012;20:729-733. doi:10.1097/JGP.0b013e31826573cf.

[88] Yiallouris A, Tsioutis C, Agapidaki E, Zafeiri M, Agouridis AP, Ntourakis D, et al. Adrenal Aging and Its Implications on Stress Responsiveness in Humans. Front Endocrinol (Lausanne) 2019;10:54. doi:10.3389/fendo.2019.00054.

[89] Yan Y-X, Xiao H-B, Wang S-S, Zhao J, He Y, Wang W, et al. Investigation of the Relationship Between Chronic Stress and Insulin Resistance in a Chinese Population. J Epidemiol 2016;26:355-360. doi:10.2188/jea.JE20150183.

[90] Yeager MP, Pioli PA, Guyre PM. Cortisol Exerts Bi-Phasic Regulation of Inflammation in Humans. Dose-Response 2011;9:332=347. doi:10.2203/dose-response.10-013.Yeager.

[91] Yeager MP, Guyre CA, Sites BD, Collins JE, Pioli PA, Guyre PM. The Stress Hormone Cortisol Enhances Interferon- $\cup-$ Mediated Proinflammatory Responses of Human Immune Cells. Anesth Analg 2018;127:556-63. doi:10.1213/ANE.0000000000003481.

[92] Manalai P, Hamilton RG, Langenberg P, Kosisky SE, Lapidus M, Sleemi A, et al. Pollen-specific immunoglobulin E positivity is associated with worsening of depression scores in bipolar disorder patients during high pollen season. Bipolar Disord 2012;14:90-8. doi:10.1111/j.1399-5618.2012.00983.x.

[93] Kelly K, Ratliff S, Mezuk B. Allergies, asthma, and psychopathology in a nationally-representative US sample. J Affect Disord 2019;251:130-5. doi:10.1016/j.jad.2019.03.026.

[94] Costa-Pinto FA, Basso AS, Britto LRG, Malucelli BE, Russo M. Avoidance behavior and neural correlates of allergen exposure in a murine model of asthma. Brain Behav Immun 2005;19:52-60. doi:10.1016/j.bbi.2004.02.005.

[95] Tonelli LH, Katz M, Kovacsics CE, Gould TD, Joppy B, Hoshino A, et al. Allergic rhinitis induces anxiety-like behavior and altered social interaction in rodents. Brain Behav Immun 2009;23:784-93. doi:10.1016/j.bbi.2009.02.017.

[96] Monda V, Villano I, Messina A, Valenzano A, Esposito T, Moscatelli F, et al. Exercise Modifies the Gut Microbiota with Positive Health Effects. Oxid Med Cell Longev 2017;2017:3831972. doi:10.1155/2017/3831972.

[97] de Souto Barreto P, Rolland Y, Vellas B, Maltais M. Association of Long-term Exercise Training With Risk of Falls, Fractures, Hospitalizations, and Mortality in Older Adults: A Systematic Review and Metaanalysis. JAMA Intern Med 2019;179:394-405. doi:10.1001/jamainternmed.2018.5406.

[98] Kraus W, Powell K, Haskell W, Janz K, Campbell W, Jakicic J, et al. Physical Activity, All-Cause and Cardiovascular Mortality, and Cardiovascular Disease. Med Sci Sports Exerc 2019;51:1270-81. doi:10.1249/MSS.00000000000

[99] Hart PD, Benavidez G, Erickson J. Meeting Recommended Levels of Physical Activity in Relation to Preventive Health Behavior and Health Status Among Adults. J Prev Med Public Heal 2017;50:10-7. doi:10.3961/jpmph.16.080.

[100] Clark A, Mach N. Exercise-induced stress behavior, gut-microbiota-brain axis and diet: a systematic review for athletes. J Int Soc Sports Nutr 2016;13:43. doi:10.1186/s12970-016-0155-6.

[101] Felger JC, Treadway MT. Inflammation Effects on Motivation and Motor Activity: Role of Dopamine. Neuropsychopharmacology 2017;42:216-41. doi:10.1038/npp.2016.143.

[102] Cui H, Kong Y, Zhang H. Oxidative Stress, Mitochondrial Dysfunction, and Aging. J Signal Transduct 2012;2012:646354. doi:10.1155/2012/646354. 
[103] Cavaliere G, Trinchese G, Penna E, Cimmino F, Pirozzi C, Lama A, et al. High-Fat Diet Induces Neuroinflammation and Mitochondrial Impairment in Mice Cerebral Cortex and Synaptic Fraction. Front Cell Neurosci 2019;13:509. doi:10.3389/fncel.2019.00509.

[104] Wilmanski T, Diener C, Rappaport N, Patwardhan S, Wiedrick J, Lapidus J, et al. Gut Microbiome Pattern Reflects Healthy Aging and Predicts Extended Survival in Humans. BioRxiv Prepr 2020. doi:10.1101/2020.02.26.966747.

[105] Hermans MAW, Lennep JER van, Daele PLA van, Bot I. Mast Cells in Cardiovascular Disease: From Bench to Bedside. Int J Mol Sci 2019;20:3395. doi:10.3390/ijms20143395.

[106] Shi MA, Shi G-P. Different Roles of Mast Cells in Obesity and Diabetes: Lessons from Experimental Animals and Humans. Front Immunol 2012;3:7. doi:10.3389/fimmu.2012.00007.

[107] Andersson CK, Mori M, Bjermer L, Löfdahl C-G, Erjefält JS. Alterations in Lung Mast Cell Populations in Patients with Chronic Obstructive Pulmonary Disease. Am J Respir Crit Care Med 2010;181:206-17. doi:10.1164/rccm.200906-0932OC.

[108] Theoharides TC, Sismanopoulos N, Delivanis D-A, Zhang B, Hatziagelaki EE, Kalogeromitros D. Mast cells squeeze the heart and stretch the gird: their role in atherosclerosis and obesity. Trends Pharmacol Sci 2011;32:534-42. doi:10.1016/j.tips.2011.05.005.

[109] Li M, Liu K, Michalicek J, Angus JA, Hunt JE, Dell'Italia LJ, et al. Involvement of chymase-mediated angiotensin II generation in blood pressure regulation. J Clin Invest 2004;114:112-20. doi:10.1172/JCI20805.

[110] Becker BF. All because of the mast cell: blocking the angiotensin receptor-1 should be better than inhibiting ACE (theoretically). Cardiovasc Res 2011;92:7-9. doi:10.1093/cvr/cvr214.

[111] Gideon A, Sauter C, Fieres J, Berger T, Renner B, Wirtz PH. Kinetics and Interrelations of the Renin Aldosterone Response to Acute Psychosocial Stress: A Neglected Stress System. J Clin Endocrinol Metab 2020;105:e762-73. doi:10.1210/clinem/dgz190.

[112] Ayada C, Toru Ü, Korkut Y. The relationship of stress and blood pressure effectors. Hippokratia 2015;19:99-108.

[113] Liu Y, Yang Y, Zhang C, Huang F, Wang F, Yuan J, et al. Clinical and biochemical indexes from 2019-nCoV infected patients linked to viral loads and lung injury. Sci China Life Sci 2020;63:364-74. doi:10.1007/s11427-020-1643-8.

[114] Mancini E, Fürst J. View: "Scorched Earth" strategy: The RAS as possible target for treating COVID-19 patients with a combination of three approved pharmaceutical agents. Figshare Prepr 2020. doi:10.13140/RG.2.2.35010.94400.

[115] Satou R, Penrose H, Navar LG. Inflammation as a Regulator of the Renin-Angiotensin System and Blood Pressure. Curr Hypertens Rep 2018;20:100. doi:10.1007/s11906-018-0900-0.

[116] El-Haggar SM, Farrag WF, Kotkata FA. Effect of ketotifen in obese patients with type 2 diabetes mellitus. J Diabetes Complications 2015;29:427-32. doi:10.1016/j.jdiacomp.2015.01.013.

[117] Sismanopoulos N, Delivanis D-A, Mavrommati D, Hatziagelaki E, Conti P, Theoharides TC. Do mast cells link obesity and asthma? Allergy 2013;68:8-15. doi:10.1111/all.12043.

[118] Piliponsky AM, Acharya M, Shubin NJ. Mast Cells in Viral, Bacterial, and Fungal Infection Immunity. Int J Mol Sci 2019;20:E2851. doi:10.3390/ijms20122851.

[119] Kritas SK, Ronconi G, Caraffa A, Gallenga CE, Ross R, Conti P. Mast Cells Contribute to CoronavirusInduced Inflammation: New Anti-Inflammatory Strategy. J Biol Regul Homeost Agents 2020;34:10.23812/20Editorial-Kritas. doi:10.23812/20-Editorial-Kritas. 
[120] Corley MJ, Sugai C, Schotsaert M, Schwartz RE, Ndhlovu LC. Comparative in vitro transcriptomic analyses of COVID-19 candidate therapy hydroxychloroquine suggest limited immunomodulatory evidence of SARS-CoV-2 host response genes. BioRxiv Prepr 2020. doi:10.1101/2020.04.13.039263.

[121] Graham AC, Temple RM, Obar JJ. Mast Cells and Influenza A Virus: Association with Allergic Responses and Beyond. Front Immunol 2015;6:238. doi:10.3389/fimmu.2015.00238.

[122] Han D, Wei T, Zhang S, Wang M, Tian H, Cheng J, et al. The therapeutic effects of sodium cromoglycate against influenza A virus H5N1 in mice. Influ Other Respir Viruses 2016;10:57-66. doi:10.1111/irv.12334.

[123] Hu Y, Jin Y, Han D, Zhang G, Cao S, Xie J, et al. Mast Cell-Induced Lung Injury in Mice Infected with H5N1 Influenza Virus. J Virol 2012;86:3347-56. doi:10.1128/JVI.06053-11.

[124] Liebler JM, Qu Z, Buckner B, Powers MR, Rosenbaum JT. Fibroproliferation and mast cells in the acute respiratory distress syndrome. Thorax 1998;53:823-9. doi:10.1136/thx.53.10.823.

[125] Overed-Sayer C, Rapley L, Mustelin T, Clarke DL. Are mast cells instrumental for fibrotic diseases? Front Pharmacol 2014;4:174. doi:10.3389/fphar.2013.00174.

[126] Keith P, Day M, Perkins L, Moyer L, Hewitt K, Wells A. A novel treatment approach to the novel coronavirus: an argument for the use of therapeutic plasma exchange for fulminant COVID-19. Crit Care 2020;24:128. doi:10.1186/s13054-020-2836-4.

[127] Dahdah A, Gautier G, Attout T, Fiore F, Lebourdais E, Msallam R, et al. Mast cells aggravate sepsis by inhibiting peritoneal macrophage phagocytosis. J Clin Invest 2014;124:4577-89. doi:10.1172/JCI75212.

[128] Ramos L, Peña G, Cai B, Deitch EA, Ulloa L. Mast Cell Stabilization Improves Survival by Preventing Apoptosis in Sepsis. J Immunol 2010;185:709-16. doi:10.4049/jimmunol.1000273.

[129] Byrne K, Sielaff TD, Michna B, Carey PD, Blocher CR, Vasquez A, et al. Increased Survival Time After Delayed Histamine and Prostaglandin Blockade in a Porcine Model of Severe Sepsis-Induced Lung Injury. Crit Care Med 1990;18:303-8. doi:10.1097/00003246-199003000-00012.

[130] Seeley EJ, Sutherland RE, Kim SS, Wolters PJ. Systemic mast cell degranulation increases mortality during polymicrobial septic peritonitis in mice. J Leukoc Biol 2011;90:591-7. doi:10.1189/jlb.0910531.

[131] Seeley EJ, Matthay MA, Wolters PJ. Inflection points in sepsis biology: from local defense to systemic organ injury. Am J Physiol - Lung Cell Mol Physiol 2012;303:L355-63. doi:10.1152/ajplung.00069.2012.

[132] Ho T-W, Huang C-T, Ruan S-Y, Tsai Y-J, Lai F, Yu C-J. Diabetes mellitus in patients with chronic obstructive pulmonary disease-The impact on mortality. PLoS One 2017;12:e0175794. doi:10.1371/journal.pone.0175794.

[133] Morgan AD, Zakeri R, Quint JK. Defining the relationship between COPD and CVD: what are the implications for clinical practice? Ther Adv Respir Dis 2018;12:1753465817750524. doi:10.1177/1753465817750524.

[134] Holt JB, Zhang X, Presley-Cantrell L, Croft JB. Geographic disparities in chronic obstructive pulmonary disease (COPD) hospitalization among Medicare beneficiaries in the United States. Int J Chron Obstruct Pulmon Dis 2011;6:321-8. doi:10.2147/COPD.S19945.

[135] Halvorsen T, Martinussen P. The Geography of Chronic Obstructive Pulmonary Disease: A PopulationBased Study of Norway. Soc Sci Med 2014;111:25-34. doi:10.1016/j.socscimed.2014.03.018.

[136] Huang X, Mu X, Deng L, Fu A, Pu E, Tang T, et al. The etiologic origins for chronic obstructive pulmonary disease. Int J Chron Obstruct Pulmon Dis 2019;14:1139-58. doi:10.2147/COPD.S203215.

[137] Jaakkola MS, Lajunen TK, Jaakkola JJK. Indoor mold odor in the workplace increases the risk of Asthma-COPD Overlap Syndrome: a population-based incident case-control study. Clin Transl Allergy 2020;10:3. doi:10.1186/s13601-019-0307-2. 
[138] Tzortzaki EG, Proklou A, Siafakas NM. Asthma in the Elderly: Can We Distinguish It from COPD? J Allergy 2011;2011:843543. doi:10.1155/2011/843543.

[139] To T, Zhu J, Larsen K, Simatovic J, Feldman L, Ryckman K, et al. Progression from Asthma to Chronic Obstructive Pulmonary Disease. Is Air Pollution a Risk Factor? Am J Respir Crit Care Med 2016;194:429-38. doi:10.1164/rccm.201510-1932OC.

[140] Veil-Picard M, Soumagne T, Vongthilath R, Annesi-Maesano I, Guillien A, Laurent L, et al. Is atopy a risk indicator of chronic obstructive pulmonary disease in dairy farmers? Respir Res 2019;20:124. doi:10.1186/s12931-019-1082-2.

[141] Eguiluz-Gracia I, Pérez-Sánchez N, Bogas G, Campo P, Rondón C. How to Diagnose and Treat Local Allergic Rhinitis: A Challenge for Clinicians. J Clin Med 2019;8:1062. doi:10.3390/jcm8071062.

[142] Baptist AP, Nyenhuis S. Rhinitis in the elderly. Immunol Allergy Clin North Am 2016;36:343-57. doi:10.1016/j.iac.2015.12.010.

[143] Wright BL, Kulis M, Guo R, Orgel KA, Wolf WA, Burks AW, et al. Food-specific IgG4 is associated with eosinophilic esophagitis. J Allergy Clin Immunol 2016;138:1190-1192.e3. doi:10.1016/j.jaci.2016.02.024.

[144] Trost B, Lucchese G, Stufano A, Bickis M, Kusalik A, Kanduc D. No human protein is exempt from bacterial motifs, not even one. Self Nonself 2010;1:328-34. doi:10.4161/self.1.4.13315.

[145] Perelmutter L, Potvin L, Phipps P. Immunoglobulin E response during viral infections. J Allergy Clin Immunol 1979;64:127-30.

[146] Yang Y, Shen C, Li J, Yuan J, Yang M, Wang F, et al. Exuberant elevation of IP-10, MCP-3 and IL-1ra during SARS-CoV-2 1 infection is associated with disease severity and fatal outcome. MedRxiv Prepr 2020. doi:10.1101/2020.03.02.20029975.

[147] Saukkonen T, Mutt SJ, Jokelainen J, Saukkonen A-M, Raza GS, Karhu T, et al. Adipokines and inflammatory markers in elderly subjects with high risk of type 2 diabetes and cardiovascular disease. Sci Rep 2018;8:1-8. doi:10.1038/s41598-018-31144-8.

[148] Brightling CE, Ammit AJ, Kaur D, Black JL, Wardlaw AJ, Hughes JM, et al. The CXCL10/CXCR3 Axis Mediates Human Lung Mast Cell Migration to Asthmatic Airway Smooth Muscle. Am J Respir Crit Care Med 2005;171:1103-8. doi:10.1164/rccm.200409-1220OC.

[149] Jing H, Liu L, Zhou J, Yao H. Inhibition of C-X-C Motif Chemokine 10 (CXCL10) Protects Mice from Cigarette Smoke-Induced Chronic Obstructive Pulmonary Disease. Med Sci Monit 2018;24:5748-53. doi:10.12659/MSM.909864.

[150] Keshavarzi F. Fungistatic effect of hydroxychloroquine, lessons from a case. Med Mycol Case Rep 2016;13:17-8. doi:10.1016/j.mmcr.2016.09.003.

[151] Gautret P, Lagier J-C, Parola P, Hoang VT, Meddeb L, Sevestre J, et al. Clinical and microbiological effect of a combination of hydroxychloroquine and azithromycin in 80 COVID-19 patients with at least a six-day follow up: an observational study Running title: Hydroxychloroquine-Azithromycin and COVID-19. Travel Med Infect Dis 2020:101663. doi:10.1016/j.tmaid.2020.101663.

[152] Shamshirian A, Hessami A, Heydari K, Alizadeh-Navaei R, Ebrahimzadeh MA, Ghasemian R, et al. Hydroxychloroquine Versus COVID-19: A Rapid Systematic Review and Meta-Analysis. MedRxiv Prepr 2020. doi:10.1101/2020.04.14.20065276.

[153] Magagnoli J, Narendran S, Pereira F, Cummings T, Hardin JW, Sutton SS, et al. Outcomes of hydroxychloroquine usage in United States veterans hospitalized with Covid-19. MedRxiv Prepr 2020. doi:/10.1101/2020.04.16.20065920. 